\title{
Evaluación microestructural de geopolímeros basados en metacaolín y fuentes alternativas de sílice expuestos a temperaturas altas
}

\section{Microstructure Assessment of Metakaolin Based-Geopolymers Produced with Alternative Silica Sources Exposed to High Temperatures}

\author{
Villaquirán-Caicedo Mónica Alejandra \\ Grupo de Materiales Compuestos (CENM) \\ Escuela de Ingeniería de Materiales \\ Universidad del Valle, Cali, Colombia \\ Correo:monica.villaquiran@correounivalle.edu.co, \\ Rodríguez Erich David \\ Grupo de Materiales Compuestos (CENM) \\ Escuela de Ingeniería de Materiales \\ Universidad del Valle, Cali, Colombia \\ Correo:erich.rodriguez@correounivalle.edu.co
}

\author{
Mejía-De Gutiérrez Ruby \\ Grupo de Materiales Compuestos (CENM) \\ Escuela de Ingeniería de Materiales \\ Universidad del Valle, Cali, Colombia \\ Correo:ruby.mejia@correounivalle.edu.co
}

Información del artículo: recibido: julio de 2013, reevaluado: octubre de 2013, aceptado: noviembre de 2013

\section{Resumen}

Los geopolímeros se obtienen a partir de la activación alcalina de minerales naturales, desechos o subproductos industriales para generar un producto con características cerámicas que presentan ventajas a nivel ambiental y con propiedades similares y en algunos casos superiores frente a materiales tradicionales. El presente estudio evalúa el efecto de la exposición a $600^{\circ} \mathrm{y}$ $1200^{\circ} \mathrm{C}$ de sistemas geopoliméricos basados en metacaolín (MK) con la utilización de activadores basados en $\mathrm{KOH}$. Se evaluó la utilización de una ceniza de cascarilla de arroz (RHA) y un humo de sílice comercial (SF) como fuentes alternativas de sílice para la preparación del activador alcalino. La exposición a las temperaturas elevadas conduce a la deshidratación de los productos formados durante la activación del MK y cambios estructurales asociados a la transformación del gel geopolimérico a estructuras cristalinas tipo leucita $\left(\mathrm{KAlSi}_{2} \mathrm{O}_{6}\right)$ y kalsilita $\left(\mathrm{KAlSiO}_{4}\right)$, lo cual promueve la densificación y reducción de la porosidad del material. La presencia de partículas de MK sin reaccionar en los materiales geopoliméricos condujo a la formación de mullita $\left(2 \mathrm{Al}_{2} \mathrm{O}_{3} \cdot \mathrm{SiO}_{2}\right)$, después del tratamiento a $1200^{\circ} \mathrm{C}$.

\section{Descriptores:}

- geopolímeros

- metacaolín

- elevadas temperaturas

- ceniza de cascarilla de arroz

- humo de sílice 


\begin{abstract}
Ceramic materials more environment friendly and with similar or even better performance than traditional ones can be produced by alkali activation of natural minerals, wastes or industrial by-products. The present study assesses the effect of exposure at $600^{\circ}$ and $1200^{\circ} \mathrm{C}$ of a MK-based geopolymers. Rice husk ash (RHA) and silica fume were modified chemically in order to obtain an alternative alkali activator. Exposure to elevated temperatures leads to dehydration of the reaction products and structural reorganization associated with the crystallization of the gel to leucite (KAl$\left.\mathrm{Si}_{2} \mathrm{O}_{6}\right)$ and kalsilite $\left(\mathrm{KAlSiO}_{4}\right)$. The structural changes associated with the thermal treatment also promote a densification and reduction of porosity. The unreacted $M K$ particles embedded into the geopolymeric gel lead to the formation of mullite $\left(2 \mathrm{Al}_{2} \mathrm{O}_{3} \cdot \mathrm{SiO}_{2}\right)$ after the thermal treatment at $1200^{\circ} \mathrm{C}$.
\end{abstract}

\section{Introducción}

Los geopolímeros se obtienen a partir de la mezcla en óptimas proporciones de un mineral basado en $\mathrm{SiO}_{2}$ y $\mathrm{Al}_{2} \mathrm{O}_{3}$ (precursor) con un agente químico (activador alcalino), el cual promueve una serie de reacciones que conducen a la formación de un producto con características cementantes. Uno de los precursores ampliamente utilizados en este tipo de tecnología son las arcillas caoliníticas tratadas térmicamente, las cuales presentan una elevada reactividad como consecuencia de su naturaleza vítrea (Davidovits, 1991; Duxson et al., 2006a; Komnitsas y Zaharaki, 2007). Los activadores son por lo general soluciones altamente alcalinas, constituidas por $\mathrm{NaOH}$ o $\mathrm{KOH}$ donde el tipo de ión alcalino tiene un efecto sobre las características y propiedades finales del producto obtenido (Haha y Saout, 2011; Duxson et al., 2005). El NaOH es el más utilizado como activador debido a que es el hidróxido alcalino con mayor disponibilidad comercial y uno de los más económicos. No obstante, la utilización de $\mathrm{K}^{+}$como ión alcalino ha tenido cierto interés debido a que el tamaño del catión podría contribuir a la mayor disolución del precursor y con ello a un mejoramiento en las propiedades reológicas de la mezcla en estado fresco (Provis, 2009), la obtención de un elevado desempeño mecánico (Phair y Deventer, 2001), así como una mayor estabilidad térmica con temperaturas de fusión hasta de $1400^{\circ} \mathrm{C}$ (Barbosa y McKenzie, 2003). El K ${ }^{+}$ puede conducir a un mayor grado de condensación comparado con el $\mathrm{Na}^{+}$cuando se suministra en las mismas condiciones (Duxson et al., 2005; Phair y Deventer, 2001). Sin embargo, en la literatura existe cierta controversia en relación con qué tipo de ión alcalino exhibe el mejor desempeño para la obtención de materiales con resistencias mecánicas más elevadas (Duxson et al., 2006a; Khale y Chaudhary, 2007).
El producto final obtenido después de la activación es un gel de aluminosilicato alcalino (N-A-S-H) con estructuras tipo $\mathrm{Q}^{4}(\mathrm{mAl})$, con $\mathrm{m}=1,2 \mathrm{o} 3$. Como producto secundario se ha identificado la presencia de diferentes estructuras de naturaleza zeolítica de bajo orden estructural (De Silva y Sagoe, 2009; Beleña et al., 2004; Juenger et al., 2011). El grado de cristalinidad y tipo de estructura zeolítica depende del tipo de activación (concentración, naturaleza y tipo del activador alcalino), tipo de precursor y las condiciones de curado (tiempo y temperatura) (De Silva y Sagoe, 2009; De Silva et al., 2007; Grutzeck y Dicola, 2004).

La incorporación de silicatos solubles en el activador alcalino, mediante la utilización de soluciones de silicato de sodio $\left(\mathrm{Na}_{2} \mathrm{SiO}_{3} \cdot \mathrm{nH}_{2} \mathrm{O}\right)$ o silicato de potasio $\left(\mathrm{K}_{2} \mathrm{SiO}_{3} \cdot \mathrm{nH}_{2} \mathrm{O}\right)$ contribuyen significativamente con la cinética de las reacciones durante la activación, el desempeño mecánico a tempranas edades y sobre las características estructurales (Komnitsas y Zaharaki, 2007). No obstante, se han empleado fuentes alternativas de sílice, provenientes de desechos y/o subproductos industriales, tales como cenizas de cascarilla de arroz (Gluth et al, 2012; Songprriyakij et al., 2010; Detphan y Chindaprasirt, 2009), humo de sílice (Bernal et al., 2012; Zivica, 2006; Rousekova et al., 1997; Bernal et al., 2011a) y micro sílice (Autef et al., 2012; Pimraksa, 2011) las cuales se modifican químicamente con soluciones de hidróxido para obtener activadores alcalinos con la presencia de $\mathrm{SiO}_{2}$ disuelta. La producción de activadores alcalinos con la presencia de estas fuentes de $\mathrm{SiO}_{2}$, además de otorgarles un valor agregado a estos subproductos, permite obtener materiales con desempeños mecánicos similares a los geopolímeros producidos con soluciones de silicatos solubles comerciales $\left(\mathrm{Na}_{2} \mathrm{SiO}_{3} \cdot \mathrm{nH}_{2} \mathrm{O}\right.$ o $\left.\mathrm{K}_{2} \mathrm{SiO}_{3} \cdot \mathrm{nH}_{2} \mathrm{O}\right)$ (Bernal et al., 2012; Zivica, 2006; Rousekova et al., 1997; Bernal et al., 2011a; Autef et al., 2012; Pimraksa, 2011; Fawer et al., 1999; Deabriges, 1982; Bernal et al., 2011b; 
Davidovits, 2002), contribuyendo a la mayor sostenibilidad ambiental de los geopolímeros, debido a que las soluciones tradicionales de silicatos solubles involucran en su producción una elevada demanda energética (temperaturas cercanas a $\operatorname{los} 1400^{\circ} \mathrm{C}$ ) y la emisión de gases invernadero (Fawer et al., 1999; Deabriges, 1982). Bernal et al. (2012) evaluó la utilización de ceniza de cascarilla de arroz (RHA) como fuente adicional de silicatos solubles en sistemas geopoliméricos basados en MK y reportó un desempeño mecánico ligeramente inferior frente a los materiales producidos con silicatos de sodio comercial; esto se atribuye a una menor disponibilidad de Si durante la activación como consecuencia de una menor reactividad de la RHA utilizada, lo cual influye en la cinética de formación del gel N-A-S-H (Bernal et al., 2012; Zivica, 2006; Rousekova et al., 1997; Bernal et al., 2011a; Autef et al., 2012; Pimraksa, 2011; Fawer et al., 1999; Deabriges, 1982; Bernal et al., 2011b).

La producción de geopolímeros involucra la utilización de temperaturas inferiores a los $100^{\circ} \mathrm{C}$ (Davidovits, 2002), lo cual presenta una ventaja a nivel técnico y económico en comparación con las cerámicas tradicionales que se utilizan actualmente en diversas aplicaciones en la ingeniería. Adicionalmente, los geopolímeros se caracterizan por exhibir una estabilidad térmica elevada, pueden conservar su integridad y propiedades mecánicas incluso a temperaturas de $800^{\circ} \mathrm{C}$ (Zuda et al., 2010; Fernández et al., 2010). Algunos autores, afirman que la exposición a temperaturas más altas $\left(1200^{\circ} \mathrm{C}\right)$ conduce a un proceso de densificación estructural y con ello el incremento de la resistencia mecánica de hasta en 30\% (Zuda et al., 2010). Las propiedades especiales como la no combustibilidad brindan un gran panorama para su utilización potencial como materiales en aplicaciones de alta tecnología (Davidovits, 2002; Zhang et al.,2012; Pacheco et al., 2008; Kong et al., 2008).

El objetivo del presente estudio es analizar el efecto de la exposición a temperaturas elevadas sobre las características estructurales de materiales geopoliméricos producidos a partir de un metacaolín (MK) activado con una mezcla de hidróxido de potasio y fuentes alternativas de sílice.

\section{Metodología experimental}

Materiales

Como precursor se empleó un metacaolín comercial MetaMax ${ }^{\circledR}(\mathrm{MK})$, con la presencia de pequeñas trazas de anatasa $\left(\mathrm{TiO}_{2}\right)$ y cuarzo $\left(\mathrm{SiO}_{2}\right)$ (figura 1), con una superficie específi- ca de $12.7 \mathrm{~m}^{2} / \mathrm{kg}$, una distribución de tamaño de partícula comprendida entre 1 y $40 \mu \mathrm{m}$ y un tamaño medio de partícula de $7.8 \mu \mathrm{m}$.

Con base en su composición química, la cual se lista en la tabla 1, el metacaolín presentó una relación molar $\mathrm{SiO}_{2} / \mathrm{Al}_{2} \mathrm{O}_{3}$ cercana a 2 . El activador alcalino se preparó mediante la mezcla de un hidróxido de potasio grado analítico de Merck y la fuente de silicatos solubles. Como fuentes de sílice se emplearon una solución de silicato de potasio $\left(\mathrm{K}_{2} \mathrm{O} \cdot \mathrm{SiO}_{3} \mathrm{H}_{2} \mathrm{O}\right)$ de productos químicos panamericanos (PQP) constituido por $13.06 \%$ de $\mathrm{K}_{2} \mathrm{O}$, $26.38 \%$ de $\mathrm{SiO}_{2}$ y $60.56 \% \mathrm{H}_{2} \mathrm{O}$, así como la utilización de un humo de sílice comercial (SF) SikaFume ${ }^{\circledR}$ de SIKA S.A. y una ceniza de cascarilla de arroz (RHA). La RHA se obtuvo mediante un tratamiento térmico en condiciones controladas (Gutiérrez et al., 2012).

Tabla 1. Composición química de los materiales

\begin{tabular}{cccc}
\hline $\begin{array}{c}\text { Componente } \\
\text { (\% en peso) }\end{array}$ & $\mathrm{MK}$ & $\mathrm{SF}$ & $\mathrm{RHA}$ \\
\hline $\mathrm{SiO}_{2}$ & 51.52 & 87.60 & 92.33 \\
$\mathrm{Al}_{2} \mathrm{O}_{3}$ & 44.53 & 0.38 & 0.18 \\
$\mathrm{TiO}_{2}$ & 1.71 & 0.01 & -- \\
$\mathrm{Fe}_{2} \mathrm{O}_{3}$ & 0.48 & 0.66 & 0.17 \\
$\mathrm{Na}_{2} \mathrm{O}$ & 0.29 & 1.26 & 0.07 \\
$\mathrm{~K}_{2} \mathrm{O}$ & 0.16 & 2.36 & 0.15 \\
$\mathrm{MgO}$ & 0.19 & 3.67 & 0.49 \\
$\mathrm{CaO}$ & 0.02 & 0.57 & 0.63 \\
Pérdida del fuego $\left(1000^{\circ} \mathrm{C}\right)$ & 1.09 & 3.49 & 2.57
\end{tabular}

Preparación de los especímenes

Se diseñaron sistemas geopoliméricos con relaciones molares totales de $\mathrm{SiO}_{2} / \mathrm{Al}_{2} \mathrm{O}_{3}$ y $\mathrm{K}_{2} \mathrm{O} / \mathrm{SiO}_{2}$ de 2.5 y 0.3 , respectivamente. $\mathrm{El}$ activador alcalino fue preparado mediante un ajuste en la proporción del hidróxido de potasio y las fuentes de sílice con el propósito de alcanzar las relaciones $\mathrm{SiO}_{2} / \mathrm{Al}_{2} \mathrm{O}_{3}$ y $\mathrm{K}_{2} \mathrm{O} / \mathrm{SiO}_{2}$ requeridas (tabla 2). La cantidad de $\mathrm{SiO}_{2}$ adicional incorporada al sistema se ajustó mediante la incorporación del SK y la fuente adicional de sílice (SF o RHA) en proporciones molares 1:1. Por su parte, el ajuste en el contenido de $\mathrm{K}_{2} \mathrm{O}$ se realizó mediante la adición de $\mathrm{KOH}$, teniendo 
en cuenta lo aportado por el SK. La relación liquido/ sólido para todas las muestras fue de 0.4 . Las soluciones activantes se agitaron a temperatura ambiente durante $24 \mathrm{~h}$ para asegurar la completa disolución de las fuentes de $\mathrm{SiO}_{2}$.

La mezcla de los componentes se llevó a cabo mecánicamente durante 7 minutos. Cada uno de los sistemas geopoliméricos se curaron a $75^{\circ} \mathrm{C}$ durante $20 \mathrm{~h}$ con humedad relativa de $\sim 90 \%$. Posteriormente, las muestras se almacenaron en una cámara húmeda a temperatura ambiente hasta completar una edad de curado de 28 días.

\section{Exposición a altas temperaturas}

Las muestras con una edad de 28 días se secaron a $60^{\circ} \mathrm{C}$ hasta lograr peso constante y se sometieron a un tratamiento térmico de $600^{\circ} \mathrm{C}$ y $1200^{\circ} \mathrm{C}$ a una velocidad de calentamiento de $1^{\circ} \mathrm{C} / \mathrm{min}$. Se llevó a cabo una isoterma de $1 \mathrm{~h}$ y un enfriamiento natural en el interior del horno. El cambio estructural de los geopolímeros por efecto de los tratamientos térmicos se evaluó tanto en los sistemas sin tratamiento térmico como en los obtenidos luego de exponerlos a dichas temperaturas utilizando diferentes técnicas instrumentales.

\section{Ensayos de caracterización estructural realizados}

Los materiales producidos, así como los expuestos a las diferentes temperaturas se evaluaron utilizando:

- Análisis termogravimétrico (TGA/DTG): se empleó un termogravímetro SDTQ600 de TA Instruments a una velocidad de calentamiento de $10^{\circ} \mathrm{C} / \mathrm{min}$ con $\mathrm{Ni}$ trógeno como gas de purga y usando crisoles de alúmina.

- Espectroscopia de infrarrojo por transformada de Fourier (FTIR): se empleó un espectrómetro de infrarrojo 100 de Perkin Elmer en modo transmitancia con una frecuencia comprendida entre 2500 y $450 \mathrm{~cm}^{-1}$. Las muestras se evaluaron con la metodología de cápsulas comprimidas de $\mathrm{KBr}$.

- Difracción de rayos X (XRD): se empleó un difractómetro X'Pert MRD de PanAnalytical usando radiación de $\mathrm{Cu} \mathrm{K} \alpha_{1}$ con un tamaño de paso de $0.020^{\circ}$ en un rango 2 entre 8 y $60^{\circ}$.

Tabla 2. Sistemas geopoliméricos producidos

\begin{tabular}{cccc}
\hline & Fuente(s) & \multicolumn{2}{c}{ Modulo } \\
\cline { 3 - 4 } Mezcla & de $\mathrm{SiO}_{2}$ & $\mathrm{SiO}_{2} / \mathrm{Al}_{2} \mathrm{O}_{3}$ & $\mathrm{~K}_{2} \mathrm{O} / \mathrm{SiO}_{2}$ \\
\hline $\mathrm{MK} / \mathrm{SK}$ & $\mathrm{SK}$ & 2.5 & 0.3 \\
$\mathrm{MK} / \mathrm{SF}$ & $\mathrm{SK}+\mathrm{SF}$ & 2.5 & 0.3 \\
$\mathrm{MK} / \mathrm{RHA}$ & $\mathrm{SK}+\mathrm{RHA}$ & 2.5 & 0.3 \\
\hline
\end{tabular}

- Microscopia electrónica de barrido (SEM): se empleó un microscopio electrónico de barrido ambiental Phenom 2005 de FEI con un voltaje de aceleración de $5 \mathrm{kV}$ y en modo de bajo vacío.

\section{Resultados y discusión}

Análisis termogravimétrico

El análisis termogravimétrico de las muestras sin tratamiento térmico (figura 1) muestra una pérdida de $\sim 6$ $7 \%$ a temperaturas inferiores a $300^{\circ} \mathrm{C}$. Las curvas DTG revelan un único pico de elevada intensidad a temperaturas comprendidas entre 25 y $300^{\circ} \mathrm{C}$. Esta pérdida se atribuye a la evaporación del agua libre contenida en los poros más grandes $\left(<100^{\circ} \mathrm{C}\right)$, al agua adsorbida en los poros del gel y el agua enlazada en la estructura KA-S-H (Duxson et al., 2007; Peigang et al., 2010a). Las muestras a $25^{\circ} \mathrm{C}$ exhiben un pico de baja intensidad y pobremente definido, centrado a $350^{\circ} \mathrm{C}$ como consecuencia de la deshidratación parcial de las estructuras zeolíticas con un bajo orden estructural (Duxson et al., 2006b), las cuales se identificaron antes en los sistemas de MK activado con $\mathrm{KOH}$. Independientemente del tipo de fuente de $\mathrm{SiO}_{2}$ empleada (SK, SF o RHA), se identificó un grado de polimerización del gel similar para cada una de las mezclas referencia $\left(25^{\circ} \mathrm{C}\right)$, teniendo en cuenta que las pérdidas de peso totales no pre-

A.

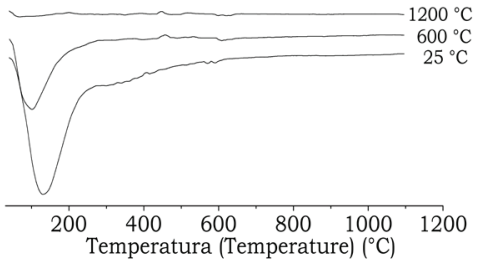

SK

B.

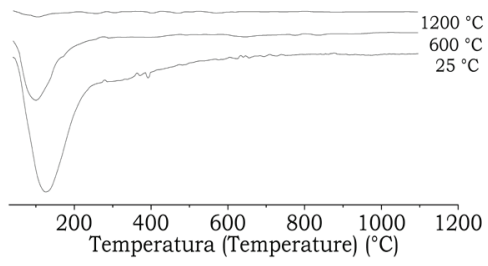

$\mathrm{SF}$

C.

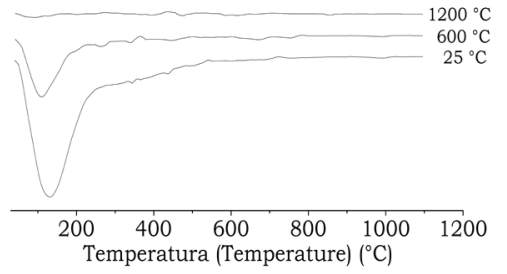

RHA

Figura 2. Análisis termogravimétrico de los sistemas geopoliméricos desde temperatura ambiente hasta $1200^{\circ} \mathrm{C}$. A. SK; B. SF; C. RHA 
sentaron diferencias significativas y fueron de $\sim 9 \%$. Los resultados DTG de las muestras expuestas a $600^{\circ} \mathrm{C}$ exhiben una reducción en las pérdidas de peso (3.7\%). La aparición del pico entre 25 y $300^{\circ} \mathrm{C}$ con una menor intensidad en las muestras que fueron expuestas a $600^{\circ} \mathrm{C}$ indica la posible rehidratación durante el enfriamiento, teniendo en cuenta que el $\mathrm{K}^{+}$presenta una alta energía de hidratación, así como la posible captura de humedad durante la preparación de la muestra. No obstante, el hombro ubicado entre 250 y $400^{\circ} \mathrm{C}$ desaparece como consecuencia de la completa deshidratación de este tipo de especies después del tratamiento.

Las curvas termogravimétricas de cada uno de los sistemas en estudio (SK, SF y RHA) expuestos a $1200^{\circ} \mathrm{C}$ exhibieron pérdidas en peso no mayores que $2 \%$, indicando un cambio estructural significativo por efecto de la exposición a dichas temperaturas, las cuales se analizarán con mayor detalle más adelante. Estos cambios estructurales, así como la ausencia de picos en $25-300^{\circ} \mathrm{C}$, indican la completa deshidratación del material y su correspondiente densificación.

\section{Difracción de rayos $X$}

En la figura 3 se muestran los difractogramas de los sistemas en estudio a las diferentes temperaturas de exposición. En los materiales sin tratamiento térmico $\left(25^{\circ} \mathrm{C}\right)$, se puede apreciar la desviación de la línea base en los ángulos $2 \theta$ para $25-35^{\circ}$, atribuido al gel geopolimérico de elevado desorden estructural, así como la identificación de los picos correspondientes a anatasa $\left(\mathrm{TiO}_{2}\right.$, Inorganic Crystal Structure Database, ICSD 154604), impureza presente en el MK, la cual no es disuelta bajo las condiciones de activación utilizadas.

La exposición de los materiales a $1200^{\circ} \mathrm{C}$ condujo a una transformación del gel K-A-S-H mediante una organización estructural por medio de la liberación del agua adsorbida en el gel (tal como se observó con los resultados de TGA) y la posterior cristalización de estructuras tipo leucita $\left(\mathrm{KAlSi}_{2} \mathrm{O}_{6}\right.$, Pattern diffraction File, PDF 00-038-1423) para cada uno de los sistemas en estudio (SK, SF y RHA). Esto condujo a una disminución significativa del grado de amorficidad, la cual se puede identificar mediante la desaparición de la desviación de la línea base en los difractogramas de los materiales expuestos a $1200^{\circ} \mathrm{C}$. La mayor intensidad de los picos atribuidos a la leucita para los sistemas SK y RHA puede atribuirse a un mayor contenido de silicatos solubles en el sistema que contribuyen a la formación de geles K-A-S-H más ricos en sílice, los cuales sufren un mayor grado de cristalización luego del tratamiento térmico. El menor grado de disolución por parte del SF conduce a un sistema con un menor contenido de sílice disponible durante la geopolimerización y por tanto la reducción en la cantidad de leucita formada durante el tratamiento.

Los picos correspondientes a anatasa del MK se identifican de la misma manera en los materiales luego de la exposición a las elevadas temperaturas. El sistema geopolimérico SK exhibió una mayor cristalización de kalsilita $\left(\mathrm{KAlSiO}_{4}\right.$, American Mineralogist Cristal Structure Database, AMCSD 1874) por efecto del tratamiento térmico. La presencia de kalsilita se atribuye a la formación de leucita, la cual se obtiene mediante tratamientos hidrotérmicos de sistemas basados en $\mathrm{Al}, \mathrm{K}$ y Si. El incremento en las temperaturas de tratamiento y la disponibilidad de un mayor contenido de Si conduce a la formación de leucita (Zhang et al., 2007). Por tanto, la presencia de kalsilita y la mayor intensidad de los picos característicos de la leucita para el sistema SK indican que la utilización de silicato de potasio como fuente de $\mathrm{SiO}_{2}$ promueve la formación de productos que presen-
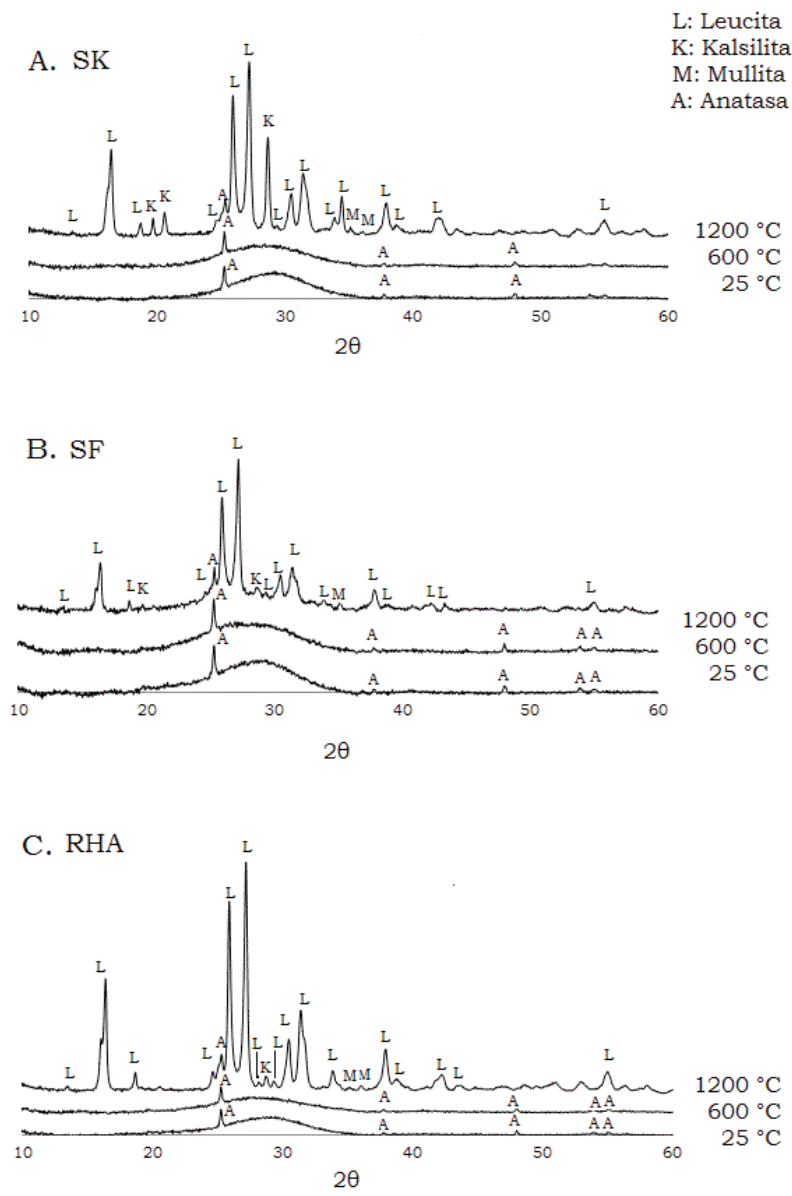

Figura 3. Difractogramas de los sistemas geopoliméricos con tratamiento térmico a $600^{\circ} \mathrm{C}$ y $1200^{\circ} \mathrm{C}$ 
tan una menor energía de activación para la cristalización de estructuras tipo leucita después de un tratamiento a $1200^{\circ} \mathrm{C}$. Estos resultados son coherentes con los estudios realizados por Tie-Song et al. (2009) y Peigang et al. (2010b), donde la formación de leucita puede quedar favorecida en sistemas con metales alcalinos de mayor peso atómico y elevados contenidos de $\mathrm{SiO}_{2}$ (Bell, 2009). Peigang et al. (2013) reportó la cristalización de leucita con un ordenamiento de largo alcance a través del tratamiento térmico de un geopolímero con relaciones $\mathrm{SiO}_{2} / \mathrm{Al}_{2} \mathrm{O}_{3}$ de 5 y $\mathrm{K}_{2} \mathrm{O} / \mathrm{SiO}_{2}$ de 0.20 a $800^{\circ} \mathrm{C}$ durante 30 minutos. La sinterización y posterior cristalización de estructuras tipo leucita puede conducir a un incremento significativo del desempeño mecánico del material, con resistencias a flexión superiores a 100 MPa (Xie et al., 2010).

\section{Infrarrojo por transformada de Fourier}

Para cada uno de los sistemas se identifica una amplia banda de gran intensidad comprendida entre 1200 y $800 \mathrm{~cm}^{-1}$ (figura 4), la cual corresponde a la tensión asimétrica de los enlaces Si-O-T (donde T corresponde a tetraedros de $\mathrm{Si}$ o Al) (Granizo et al., 2007; Heah et al., 2013; Elimbi et al., 2011). Esta banda Si-O-T en el sistema SF se encuentra a longitudes ligeramente mayores $\left(1016 \mathrm{~cm}^{-1}\right)$ en comparación con lo exhibido por RHA y SK (cuyas señales están ubicadas a $1007 \mathrm{~cm}^{-1}$ y 1012 $\mathrm{cm}^{-1}$, respectivamente), lo cual podría atribuirse a la presencia de Si cristalina que no se ha disuelto por el entorno alcalino (Bernal et al., 2012). Esta banda independientemente del tipo de activador utilizado, así como la temperatura de exposición se centra a $1015 \mathrm{~cm}^{-1}$, la cual se asigna al modo de vibración de los enlaces $\mathrm{Si}$-O-Si. Las señales correspondientes a la vibración por flexión de los enlaces Si-O-Si se detectan a 460 $\mathrm{cm}^{-1}$ y su correspondiente vibración asimétrica está ubicada a $800 \mathrm{~cm}^{-1}$ contribuyendo con la intensidad del pico Si-O-T. La señal para los sistemas activados con las diferentes fuentes de sílice sin haber sido expuestos a altas temperaturas (espectros de la figura 4 a) presenta señales entre 590-595 $\mathrm{cm}^{-1}$ atribuidas a las vibraciones por tensión simétrica de Si-O-Al (Panagiotopoulou et al., 2006) y la señal identificada a $\sim 860 \mathrm{~cm}^{-1}$ corresponde a la vibración por tensión asimétrica del Al coordinado tetraédricamente presente en el gel formado y el cual es característico en este tipo de materiales (Granizo et al., 2007). Las señales identificadas a $~ 710 \mathrm{~cm}^{-1}$ corresponden al estiramiento de los enlaces $\mathrm{Si}-\mathrm{O}-\mathrm{Si}(\mathrm{Al})$ cuyas intensidades evidencian un elevado grado de sustitución de $\mathrm{Al}$ en el gel rico en $\mathrm{Si}$, característico de la formación de polímeros amorfos (Granizo et al., 2007; Heah et al., 2013; Elimbi et al., 2011). De igual manera, con esta condición $\left(25^{\circ} \mathrm{C}\right)$ se aprecia una banda ubicada a $\sim 1650 \mathrm{~cm}^{-1}$ correspondiente a los modos de vibración por tensión de las bandas de $\mathrm{H}-\mathrm{OH}$ en las moléculas de agua asociadas a los productos de reacción (Elimbi et al., 2011).

La exposición de los materiales a las temperaturas en estudio no condujo desplazamientos de la banda Si$\mathrm{O}-\mathrm{T}$, solo se identificó un leve incremento en su intensidad y amplitud. Esto indica que no se presentaron cambios significativos en la distancia y el ángulo de los enlaces Si-O-T. Por otra parte, la mayor intensidad de la señal Si-O-T para los sistemas SK y RHA a temperatura ambiente (figura 4a) indica la presencia de estructuras con un mayor grado de desorden y menor empaquetamiento de los tetraedros, lo cual favorece una mayor movilidad del agua a través de los espacios dejados y, por tanto, la posterior cristalización de estructuras tipo leucita, cuyos picos característicos en el XRD (figura 3) presentan una mayor intensidad para los sistemas SK y RHA después del tratamiento a $1200^{\circ} \mathrm{C}$.
A. $25^{\circ} \mathrm{C}$

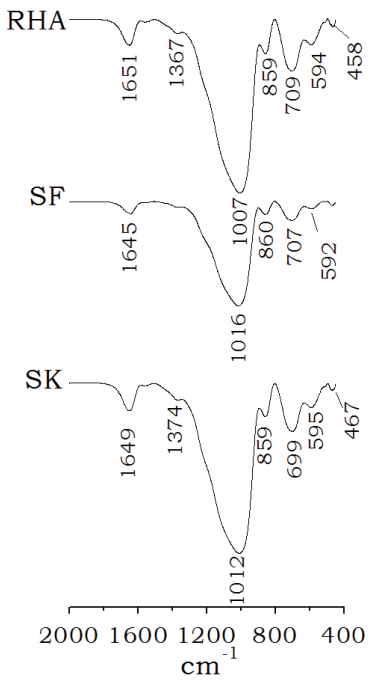

Figura 4. Espectroscopia de infrarrojo con transformada de Fourier, a) muestras de referencia sin tratamiento térmico, b) muestras expuestas a $600^{\circ} \mathrm{C}$, c) muestras expuestas a $1200^{\circ} \mathrm{C}$ 
Las señales ubicadas a $1650 \mathrm{~cm}^{-1}$ (modos de vibración del $\mathrm{H}-\mathrm{OH}$ ) disminuyen su intensidad progresivamente conforme se incrementa la temperatura de exposición de 600 a $1200^{\circ} \mathrm{C}$, lo cual es coherente con los resultados obtenidos previamente en TGA. Con el tratamiento térmico a $600^{\circ} \mathrm{C}$ las bandas ubicadas entre 590 y $860 \mathrm{~cm}^{-1}$ desaparecen y se identifican nuevas señales de elevada intensidad a $\sim 716 \mathrm{~cm}^{-1}$, para cada una de las muestras y el aumento en la intensidad de la banda a $\sim 470 \mathrm{~cm}^{-1}$.

La progresiva transformación del gel geopolimérico a una estructura tipo leucita trae consigo un colapso estructural como consecuencia de la reorganización de los cationes, cambio en los tetraedros de $\mathrm{Al}$ y por tanto la posterior desaparición de las señales a $\sim 860 \mathrm{~cm}^{-1}$ que estaban presentes en los geopolímeros a $25^{\circ} \mathrm{C}$. Este reordenamiento microestructural se evidencia en los espectros de FTIR de las muestras expuestas a $1200^{\circ} \mathrm{C}$ (figura 3c) a partir de la aparición de los picos a 707, 631, 539 y 452 $\mathrm{cm}^{-1}$, los cuales son característicos de la leucita (Sadtler Spectral Databases BIORAD, mineral number 000367) y cuya intensidad es mayor para el sistema SK, seguido por RHA. Estos resultados son coherentes con los obtenidos previamente en XRD (figura 3). Las cerámicas basadas en leucita exhiben una estabilidad a temperatura ambiente elevada y una expansión térmica alta (15.1$31 \times 10^{-60} \mathrm{C}$ ) para su potencial utilización en una variedad de aplicaciones en la unión de metales, especialmente las relacionadas con restauraciones dentales (Mackert y Evans, 1991).

\section{Microscopía electrónica de barrido}

En las micrografías de cada uno de los sistemas geopoliméricos sin tratamiento térmico (figuras $5 \mathrm{a}, \mathrm{d}$ y g) se puede apreciar la presencia de partículas de MK sin reaccionar y embebidas en el gel (tal como se indica en cada micrografía) y la obtención de un material de elevada porosidad. Conforme se incrementa la temperatura de exposición se observa de manera general una densificaciónestructural como consecuencia de la deshidratación de los productos formados y los cambios estructurales generados. Para los sistemas SF y RHA expuestos a $1200^{\circ} \mathrm{C}$ (figuras $5 f$ y $5 i$, respectivamente) se identifica el crecimiento de grietas, las cuales se generan por efecto de los esfuerzos de contracción capilar durante la deshidratación y deshidroxilación del material. La presencia de dichas grietas en menor proporción para el caso de SK puede atribuirse a una mejor distribución de la pérdida de agua en la estructura. Estas grietas pueden conducir a una disminución de las resistencias mecánicas, las cuales pueden reducirse a través de la incorporación de partículas con una elevada estabilidad térmica, tales como alúmina o circonio (Kamseu et al., 2010), mediante la inclusión de fibras cerámicas o la aplicación de presión durante los procesos de fabricación del geopolímero (Xie et al., 2019). Los poros de gran tamaño identificados en las muestras expuestas a $1200^{\circ} \mathrm{C}$ se atribuyen a los espacios que quedan como consecuencia de la evaporación del agua y la retracción posterior del material.

Las micrografías obtenidas no revelan la presencia de los cristales de leucita o kalsilita, identificados a través de XRD, probablemente debido a que pueden estar dispersos y embebidos en el gel sinterizado. El crecimiento e identificación de dichas estructuras a través de SEM se favorece conforme se incrementan los tiempos de exposición a temperaturas superiores a los $1000^{\circ} \mathrm{C}$ (Bell et al., 2009).

La estabilidad térmica, resistencia a la ignición, así como las elevadas resistencias mecánicas exhibidas por
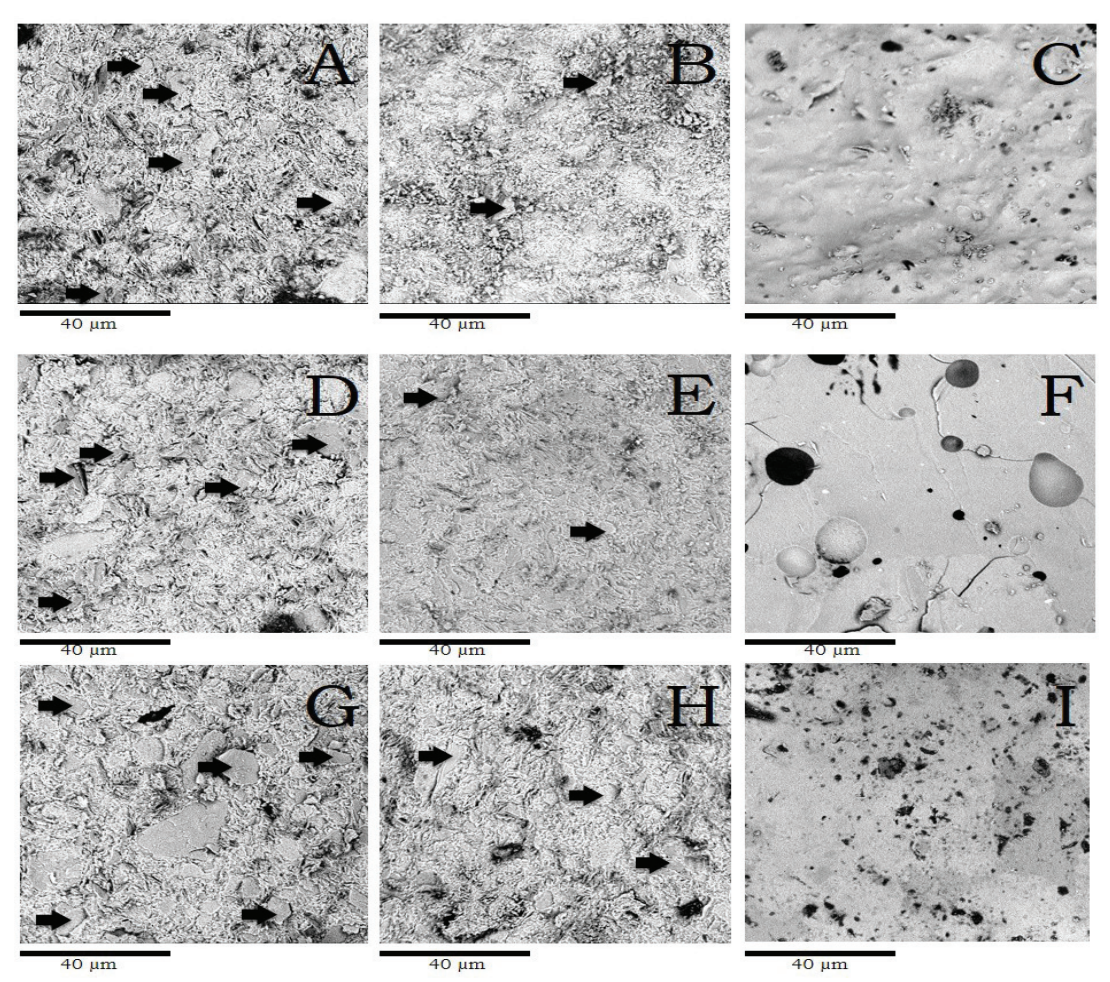

Figura 5. Micrografías de los sistemas geopoliméricos, a) SK, b) SK-600, c) SK-1200, d) $\mathrm{SF}$, e) SF- $600^{\circ}$, f) SF- $1200^{\circ}$, g) RHA, h) RHA- $600^{\circ}$, i) $\mathrm{RHA}-1200^{\circ}$ 
materiales geopoliméricos permite su utilización potencial en la industria de la construcción y transporte como materiales de revestimiento, para protección contra el fuego tanto de estructuras cerámicas como metálicas. Asimismo, estos materiales pueden emplearse como materiales de uso en la industria de procesamientos de materiales no ferrosos como moldes de fundición. Los compuestos basados en geopolímeros reforzados con fibras de carbono o fibras poliméricas pueden emplearse en plataformas marinas, barcos, así como en la construcción de paneles en aviones comerciales en donde no es posible el empleo de compuestos de matriz orgánica debido a su baja resistencia a la ignición.

\section{Conclusiones}

Los resultados obtenidos revelan que la utilización de diferentes fuentes de sílice en la producción de activadores alcalinos tiene un efecto sobre el comportamiento de los sistemas geopoliméricos cuando se someten a temperaturas elevadas. El gel K-A-S-H, principal producto de reacción obtenido tras la activación del MK, sufre una transformación después de exponer los especímenes a $1200^{\circ} \mathrm{C}$, esto ocurre como consecuencia de una reorganización microestructural por la deshidratación de los productos y la cristalización posterior de leucita. No obstante, la utilización de fuentes de sílice con un grado de reactividad elevado, como el SK y RHA, presentan una mayor disponibilidad de sílice disuelta para la formación de leucita. Se identificó la presencia de kalsilita como producto intermedio en el sistema SK después del tratamiento térmico. La producción de leucita utilizando un geopolímero producido a partir de un metacaolin (MK) activado con $\mathrm{K}^{+}$, exhibe una gran variedad de beneficios técnicos y económicos para su utilización potencial en una variedad de aplicaciones de alta tecnología.

\section{Agradecimientos}

Los autores agradecen a la Universidad del Valle (Cali, Colombia), al Centro de Excelencia en Nuevos Materiales (CENM), al Instituto Colombiano para el Desarrollo de la Ciencia y Tecnología "Francisco José de Caldas" (Colciencias) y al Banco Mundial en el marco del Contrato RC No. 0372- 2012 por el apoyo recibido dentro de la Convocatoria 528 de 2011 para la formación de doctores.

\section{Referencias}

Autef A., Joussein E., Gasgnier G., Rossignol S. Role of the Silica Source on the Geopolymerization Rate. J. Non. Cryst. Solids, volumen 358 (número 21), octubre 2012: 2886-2893.
BarbosaV.F.F. y MacKenzie K.J.D. Synthesis and Thermal Behaviour of Potassium Sialate Geopolymers. Mater. Lett., volumen 57 (números 9-10), febrero 2010: 1477-1482.

Beleña I., Tendero M.J.L., Tamayo E.M., Vie D. Cerámica y vidrio para la obtención de material geopolimérico, volumen 200, 2004, pp. 569-572.

Bell J.L., Driemeyer P.E., Kriven W.M. Formation of Ceramics from Metakaolin-Based Geopolymers: Part I-Cs-Based Geopolymer. J. Am. Ceram. Soc., volumen 92 (número 1), enero 2009: 1-8.

Bernal S.A., Rodríguez E.D., Mejía de Gutiérrez R., Gordillo M., y Provis J.L. Mechanical and Thermal Characterisation of Geopolymers Based on Silicate-Activated Metakaolin/Slag Blends. J. Mater. Sci., abril 2011a.

Bernal S.A., Provis J.L., Rose V., Mejía-De Gutierrez R. Evolution of Binder Structure in Sodium Silicate-Activated Slag-Metakaolin Blends. Cem. Concr. Compos., volumen 33 (número 1), enero 2011b: 46-54.

Bernal S., Rodríguez E., Mejía de Gutierrez R., Provis J., y Delvasto S. Activation of Metakaolin/Slag Blends Using Alkaline Solutions Based on Chemically Modified Silica Fume and Rice Husk Ash. Waste and Biomass Valorization, volumen 3, (número 1), 2012: 99-108, 2012.

Davidovits J. Geopolymers: Inorganic Polymeric New Materials. J. Therm. Anal., volumen 37 (número 8), 1991: 1633-1656.

Davidovits P.J. 30 Years of Successes and Failures in Geopolymer Applications. Market Trends and Potential Breakthroughs, 2002, pp. 1-16.

Deabriges J. Process for the Manufacture os Sodium Silicate, 4, 336, 2351982.

De Gutiérrez R.M., Ospina M., Salas A., Delvasto S. Procedimiento para la obtención de una puzolana por tratamiento térmico y físico y producto obtenido a partir del mismo, contrato/registro: 08-061404-00000-0000. Resolución: 560502012.

De Silva P. y Sagoe-Crenstil K. The Role of $\mathrm{Al} 2 \mathrm{O}$ 3, $\mathrm{SiO} 2$ and $\mathrm{Na}$ $2 \mathrm{O}$ on the Amorphous. Crystalline Phase Transformation in Geopolymer Systems, volumen 45 (número 4), 2009: 63-71, 2009.

De Silva P., Sagoe-Crenstil K., Sirivivatnanon V. Kinetics of Geopolymerization: Role of $\mathrm{Al} 2 \mathrm{O} 3$ and $\mathrm{SiO} 2$. Cem. Concr. Res., volumen 37 (número 4), abril 2007: 512-518.

Detphan S. y Chindaprasirt P. Preparation of Fly Ash and Rice Husk Ash Geopolymer. Int. J. Miner. Metall. Mater., volumen 16 (número 6), 2009: 720-726.

Duxson P., Lukey G.C., Deventer J.S.J. Physical Evolution of NaGeopolymer Derived from Metakaolin up to $1000^{\circ} \mathrm{C}$. J. Mater. Sci., volumen 42 (número 9), febrero 2007: 3044-3054.

Duxson P., Fernández-Jiménez J.L., Provis G.C., Lukey A.P., Deventer J.S.J. Geopolymer Technology: the Current State of the Art, J. Mater. Sci., volumen 42 (número 9), diciembre 2006a: 2917-2933.

Duxson P., Lukey G.C., Separovic F., Van Deventer J.S.J. Effect of Alkali Cations on Aluminum Incorporation in Geopolymeric 
Gels.Ind. Eng. Chem. Res., volumen 44 (número 4), enero 2005: 832-839.

DuxsonP., Lukey G.C., Van-Deventer J.S.J. Thermal Evolution of Metakaolin Geopolymers: Part 1-Physical evolution. J. Non. Cryst. Solids, volumen 352 (números 52-54), diciembre 2006b: 5541-5555.

Elimbi A., Tchakoute H.K., Njopwouo D. Effects of Calcination Temperature of Kaolinite Clays on the Properties of Geopolymer Cements. Constr. Build. Mater., volumen 25 (número 6), junio 2011: 2805-2812.

Fawer M., Concannon M., Rieber W. Life Cycle Inventories for the Production of Sodium Silicates. Int. J. Life Cycle Asses, volumen 4 (número 4), 1999: 201-212.

Fernández-Jiménez A., Pastor J.Y., Martín A., Palomo A. HighTemperature Resistance in Alkali-Activated Cement. J. Am. Ceram. Soc., volumen 93 (número 10), octubre 2010: 3411-3417.

Gluth G.J.G., Lehmann C., Rübner K., Kühne H. Geopolymerization of a Silica Residue from Waste Treatment of Chlorosilane Production. Mater. Struct., noviembre 2012.

Granizo M.L., Blanco-Varela M.T., Martínez-Ramírez S. Alkali Activation of Metakaolins: Parameters Affecting Mechanical, Structural and Microstructural Properties. J. Mater. Sci., volumen 42 (número 9), enero 2007: 2934-2943.

Grutzeck M., Kwan S., Dicola M. Zeolite Formation in Alkali-Activated Cementitious Systems. Cem. Concr. Res., volumen 34 (número 6), junio 2004: 949-955.

Haha M.B., Le-Saout G., Winnefeld F., Lothenbach B. Influence of Activator Type on Hydration Kinetics, Hydrate Assemblage and Microstructural Development of Alkali Activated BlastFurnace Slags. Cem. Concr. Res., volumen 41 (número 3), marzo 2011: 301-310.

Heah C.Y., Kamarudin H., Mustafa A.M., Bakri Al, Bnhussain M., Luqman M., Khairul-Nizar I., Ruzaidi C.M., Liew Y.M. Kaolin-Based Geopolymers with Various $\mathrm{NaOH}$ Concentrations. Int. J. Miner. Metall. Mater., volumen 20 (número 3), marzo 2013: 313-322.

Juenger M.C.G., Winnefeld F., Provis J.L., Ideker J.H. Advances in Alternative Cementitious Binders. Cem. Concr. Res., volumen 41 (número 12), diciembre 2012: 1232-1243.

Khale D. y Chaudhary R. Mechanism of Geopolymerization and Factors Influencing its Development: a Review. J. Mater. Sci., volumen 42 (número 3), enero 2007: 729-746.

Kamseu E., Rizzuti A., Leonelli C., Perera D. Enhanced Thermal Stability in K2O-Metakaolin-Based Geopolymer Concretes by Al2O3 and SiO2 Fillers Addition. J. Mater. Sci., volumen 45 (número 7), enero 2010: 1715-1724.

Komnitsas K. y Zaharaki D. Geopolymerisation: A Review and Prospects for the Minerals Industry. Miner. Eng., volumen 20 (número 14), noviembre 2007: 1261-1277.

Kong D.L.Y., Sanjayan Æ.J.G., Sagoe-Crentsil K. Factors Affecting the Performance of Metakaolin Geopolymers Exposed to Elevated Temperatures. J. Mater. Sci. (full set), 2008: 824-831.
Mackert J.R. y Evans A.L. Quantitative X-Ray Diffraction Determination of Leucite Thermal Instability in Dental Porcelain. J. Am. Ceram. Soc., volumen 74 (número 2), febrero 1991: 450453.

Pacheco-Torgal F., Castro-Gomes J.P., Jalali S. Adhesion Characterization of Tungsten Mine Waste Geopolymeric Binder. Influence of OPC Concrete Substrate Surface Treatment. Constr. Build. Mater., volumen 22 (número 3), marzo 2008: 154-161.

Panagiotopoulou C., Kontori E., Perraki T., Kakali G. Dissolution of Aluminosilicate Minerals and by-Products in Alkaline Media. J. Mater. Sci., volumen 42 (número 9), diciembre 2006: 2967-2973.

Peigang He., Jia D., Wang M., Zhou Y. Effect of Cesium Substitution on the Thermal Evolution and Ceramics Formation of Potassium-Based Geopolymer. Ceram. Int., volumen 36 (número 8), diciembre 2010a: 2395-2400

Peigang He., Jia D., Lin T., Wang M., Zhou Y. Effects of High-Temperature Heat Treatment on the Mechanical Properties of Unidirectional Carbon Fiber Reinforced Geopolymer Composites. Ceram. Int., volumen 36 (número 4), mayo 2010b: 1447-1453.

Peigang He., Jia D., Wang S. Microstructure and Integrity of Leucite Ceramic Derived from Potassium-Based Geopolymer Precursor. J. Eur. Ceram. Soc., volumen 33 (número 4), abril 2013: 689-698.

Phair J.W. y Van-Deventer J.S.J. Effect of Silicate Activator $\mathrm{pH}$ on the Leaching and Material Characteristics of Waste-Based Inorganic Polymers. Miner. Eng., volumen 14 (número 3), marzo 2001: 289-304.

Pimraksa K., Chindaprasirt P., Rungchet A., Sagoe-Crentsil K., Sato T. Lightweight Geopolymer Made of Highly Porous Siliceous Materials with Various $\mathrm{Na2O} / \mathrm{Al} 2 \mathrm{O} 3$ and $\mathrm{SiO} 2 / \mathrm{Al} 2 \mathrm{O} 3$ Ratios. Mater. Sci. Eng. A, volumen 528 (número 21), agosto 2011: 6616-6623

Provis J.L. Activating Solution Chemistry for Geopolymers, en: Geopolymers: Structure, Processing, Properties and Industrial Applications, Provis J.L.y Van-Deventer J.S.J., Eds. Abingdon, UK, Woodhead Publishing, 2009, pp. 50-71.

Rousekova I., Bajza A., y Zivica V. Silica Fume-Basic Blast Furnace Slag Systems Activated by an Alkali Silica Fume Activator. Science (80-. )., volumen 27 (número 12), 1997: 1825-1828.

Songpiriyakij S., Kubprasit T., Jaturapitakkul C., Chindaprasirt P. Compressive Strength and Degree of Reaction of Biomassand Fly Ash-Based Geopolymer. Constr. Build. Mater., volumen 24 (número 3), marzo 2010: 236-240

Tie-Song L.I.N., De-Chang J.I.A., Pei-Gang H.E., Mei-Rong W. Thermal-Mechanical Properties of Short Carbon Fiber Reinforced Geopolymer Matrix Composites Subjected to Thermal Load, 2009, pp. 881-886.

Xie N., Bell J.L., Kriven W.M. Fabrication of Structural Leucite Glass-Ceramics from Potassium-Based Geopolymer Precursors. J. Am. Ceram. Soc., volumen 93 (número 9), abril 2010: 2644-2649. 
Zhang Z., Yao X., Wang H. Potential Application of Geopolymers as Protection Coatings for Marine Concrete III. Field experiment. Appl. Clay Sci., volumen 67-68, octubre 2012: 57-60.

Zhang Y., M.Lv, Chen D., Wu J. Leucite Crystallization Kinetics with Kalsilite as a Transition Phase. Materials Letters, volumen 61 (número 14), 2007: 2978-298.

Zivica V. Effectiveness of New Silica Fume Alkali Activator. Cem. Concr. Compos., volumen 28 (número 1), enero 2006: 21-25.

Zuda L., Drchalová J., Rovnaník P., Bayer P., Keršner Z., Černý R. Alkali-Activated Aluminosilicate Composite with Heat-Resistant Lightweight Aggregates Exposed to High Temperatures: Mechanical and Water Ttransport Properties. Cem. Concr. Compos., volumen 32 (número 2), febrero 2010: 157-163.

\section{Este artículo se cita:}

\section{Citación estilo Chicago}

Villaquirán-Caicedo, Mónica Alejandra, Erich David Rodríguez, Ruby Mejía De Gutiérrez. Evaluación microestructural de geopolímeros basados en metacaolín y fuentes alternativas de sílice expuestos a temperaturas altas. Ingeniería Investigación y Tecnología, XVI, 01 (2015): 113-122.

\section{Citación estilo ISO 69}

Villaquirán-Caicedo M.A., Rodríguez E.D., Mejía de Gutiérrez R. Evaluación microestructural de geopolímeros basados en metacaolín y fuentes alternativas de sílice expuestos a temperaturas altas. Ingeniería Investigación y Tecnología, volumen XVI (número 1), enero-marzo 2015: 113-122.

\section{Semblanza de los autores}

Mónica Alejandra Villaquirán-Caicedo. Es ingeniera de materiales por la Universidad del Valle (Cali, Colombia) y master en ingeniería con énfasis en ingeniería de materiales por la misma institución. Es miembro activo del grupo materiales compuestos, categoría A1-2014 en colciencias; grupo al cual ha estado vinculada como joven investigador y asistente de investigación participando en diversos proyectos de la línea de investigación: "Aprovechamiento de desechos y residuos sólidos industriales". Ha participado como asistente de docencia en cursos de materiales compuestos y propiedades de los materiales en el programa académico de ingeniería de materiales. Actualmente es candidata a doctor en ingeniería, programa que adelanta con apoyo de beca doctoral de colciencias.

Erich David Rodríguez. Ingeniero de materiales por la Universidad del Valle, cuenta con dos maestrías en ingeniería relacionadas con materiales (España y Colombia) y un doctorado en ingeniería de la construcción por la Universitat Politècnica de València.

Ruby Mejía De Gutièrrez. Química por la Universidad del Valle con un master en ciencias químicas por la Universidad del Valle. Es doctora en ciencias químicas (materiales) por la Universidad Complutense de Madrid. Es directora del grupo de investigación de materiales compuestos de la Universidad del Valle desde su conformación (1986). Fue directora de la Escuela de Ingeniería de Materiales (Universidad del Valle) durante los años 2000 y 2006. Actualmente es profesor titular de la Escuela de Ingeniería de Materiales en la Universidad del Valle y coordinadora del programa de posgrado en la misma unidad académica. 